\title{
Management et performances des périmètres irrigués dans les pays en développement Présentation de l'Institut international de management de l'irrigation
}

\author{
Jean Verdier \\ Responsable des activités de l'IIMI au Maroc \\ 461, avenue Hassan II, Rabat Akkari (Maroc) [Téléphone \& télécopie : (212-7) 691101 ]
}

\section{Les défis de l'agriculture irriguée dans le Tiers Monde}

Bien que ne couvrant que le sixième des terres cultivées de la planète, les 250 millions d'hectares d'agriculture irriguee contribuent à plus du tiers de la production agricole mondiale, proportion atteignant $55 \%$ pour les produits alimentaires de base tels que le blé et le riz. Les trois quarts de ces superficies irriguées se trouvent dans des pays en développement. Vingt-trois d'entre eux (quinze en Asie, cinq en Amérique latine et trois en Afrique du Nord) irriguent plus d'un million d'hectares et les deux pays plus peuplés, la Chine et l'Inde, comptent chacun environ quarante-cinq millions d'hectares irrigués. Cette importance de l'irrigation pour la satisfaction des besoins alimentaires devrait encore croître dans les années futures, la Banque mondiale et le PNUD considérant que $80 \%$ des besoins alimentaires nouveaux des trois milliards d'êtres humains supplémentaires attendus d'ici à l'an 2025 ne pourront être satisfaits que par des productions irriguées. Sur ces bases, l'IIMI estime que, même en ne tablant que sur une croissance lente de la consommation alimentaire individuelle dans les pays en développement $(1,0 \%$ par an en Amérique latine et dans les pays arabes, $0,6 \%$ en Asie de l'Est et du Sud et $0,2 \%$ en Afrique sub-saharienne), l'augmentation annuelle des productions irriguées devrait être de $3,5 \%$ et atteindre $4 \%$ en certains endroits.

Or la prolongation des tendances de la dernière décennie indique que, parmi les pays en développement, seuls ceux
d'Asie de l'Est et du Sud devraient pouvoir atteindre ces objectifs sans nouvelle aide extérieure. Encore faudrait-il que cette tendance ne s'y infléchisse pas du fait d'une dégradation déjà visible de la qualité des ressources en eaux et de la concurrence de plus en plus vive pour son utilisation entre l'agriculture, les besoins domestiques et l'industrie; ainsi, et pour ne citer qu'un exemple, les besoins de la ville de Pékin amputeront les fournitures d'eau aux agriculteurs environnants de $30 \%$ à $40 \%$ d'ici la fin du siècle. Enfin, il faut mentionner que, même si elles se sont considérablement améliorées depuis vingt ans, les économies agricoles de l'Asie méridionale et orientale restent fragiles et sont en particulier menacées d'effondrement à terme si ne sont pas freinées leurs propensions actuelles à la surproduction structurelle de riz.

Malheureusement, à l'exception de l'Amérique latine, la quasi-totalité des pays en développement ne disposent que de peu de ressources en eau renouvelables pour alimenter leurs périmètres irrigués. $\mathrm{Si}$ cela semble évident pour les zones arides à semi-arides allant de l'Afrique sahélienne à l'Asie de l'Ouest, c'est également vrai de pays de mousson où, du fait de la forte densité des populations, les ressources renouvelables par habitant n'atteignent que la moitié de la moyenne mondiale $\left(3370 \mathrm{~m}^{3}\right.$ par an contre $7690 \mathrm{~m}^{3}$ par an). Aussi, même si toutes les ressources en eau n'ont pas encore été mobilisées et s'il reste possible d'aménager de nouveaux périmètres, une amélioration, même très lente, de l'alimentation des populations du Tiers Monde implique un accroissement considérable de la productivité

\section{Management and performances of irrigation systems in developing countries Presentation of the International Irrigation Management Institute}

Irrigated agriculture, which provides more than half the basic world food, should contribute to $80 \%$ of the needs generated by the population growth between now and 2025. This implies an important sustainable improvement of the performances of irrigation systems in developing countries. IIMI aims to develop action researches to foster the improvement of irrigation performances and, despite its young age, its impacts are visible in several countries. However, efforts in that direction should be considerably increased to meet the demographic challenge of the future decades. 
agricole de l'eau, c'est-à-dire de la quantité de matière agricole produite par unité de volume d'eau mobilisé. Ainsi, au Maroc, le Conseil supérieur de l'eau a évalué que les volumes d'eau annuellement disponibles pour l'irrigation chuteront de $750 \mathrm{~m}^{3}$ par habitant actuellement à $330 \mathrm{~m}^{3}$ en 2020 et ceci malgré une augmentation de la superficie irriguée de 1,3 à 1,6 million d'hectares.

Mais, pour être durable, toute amélioration de la productivité agricole de l'eau devra respecter l'environnement, la santé des agriculteurs et des consommateurs et la justice sociale.

Enfin, cette recherche opiniâtre d'une meilleure productivité devra se faire dans le contexte des profonds réajustements structurels en cours dans la grande majorité des pays en développement. Or, s'ils sont porteurs de grands espoirs à terme, ces réajustements commencent souvent par déstructurer profondément, et sans doute pour plusieurs années, l'organisation sociale de la production agricole irriguée lorsqu'ils touchent à la distribution de l'eau ou d'autres intrants, à la commercialisation des productions, à la structure des exploitations ou aux modes d'association des agriculteurs.

Ce constat pourrait être affligeant et démoralisant si la productivité agricole de l'eau n'était pas aussi faible dans tous les pays en développement et si donc la marge de gain potentiel n'y était aussi considérable pour peu qu'une attention suffisante soit apportée au problème.

Mais les causes de ces médiocres performances universelles actuelles sont très variables d'une région à l'autre. Suivant le cas, le maillon le plus faible de la chaîne amenant l'eau de son lieu de mobilisation (barrage le plus souvent) aux racines des plantes peut être :

- le transport entre le lieu de prélèvement et le lieu d'utilisation, un fort pourcentage des volumes prélevés se perdant par infiltration, par fuites ou dans des décharges du fait d'un mauvais entretien ou de régulations inadaptées (cas fréquent en Asie du Sud);

- la distribution aux agriculteurs lorsque des déséquilibres importants entre les volumes alloués entre l'amont et l'aval des réseaux conduisent simultanément à des gaspillages et à des pénuries (cas presque systématique dans les grandes plaines de l'est du Pakistan et du nord-ouest de l'Inde); - l'application de l'eau à la parcelle, (i) soit parce que les techniques utilisées imposent des doses pléthoriques (cas paradoxalement courant dans les systèmes traditionnels des zones arides et semi-arides de l'Afrique du Nord et de l'Asie de l'Ouest), (ii) soit parce que l'irrigation est mal conduite par méconnaissance ou par manque d'intérêt (ouvriers vacataires), (iii) soit parce que l'eau n'est pas appliquée au moment optimum du fait d'un suivi incorrect du bilan hydrique de la parcelle, d'une mauvaise connaissance des conséquences sur la production utile d'un stress hydrique ou, tout simplement, par indisponibilité de maind'œuvre aux moments opportuns.

Cependant, dans la plupart des cas, il semble parfaitement concevable qu'un meilleur management accompagné d'améliorations physiques modestes permette de doubler la productivité de l'eau. Ainsi l'efficience des transports d'eau en canaux dépasse rarement les $20 \%$ dans le Penjab pakistanais mais est en moyenne de $60 \%$ au Mexique et atteint $80 \%$ sur certains périmètres marocains. Toujours au Pakistan, les rapports entre les volumes délivrés en amont et en aval des tertiaires (équité) sont couramment de trois à quatre et il arrive que l'eau n'atteigne pas l'extrémité aval tandis que la distribution est presque partout parfaitement équitable dans les grands périmètres marocains. Pour ce qui est de l'application à la parcelle, une comparaison faite durant la campagne 1985-86 dans le périmètre du Tadla, au Maroc, a montré que la productivité agricole moyenne de l'eau obtenue par les agriculteurs était de $0,7 \mathrm{~kg} / \mathrm{m}^{3}$ pour le blé et de $2,8 \mathrm{~kg} / \mathrm{m}^{3}$ (production brute) pour la luzerne alors que des productivités de respectivement $1,75 \mathrm{~kg} / \mathrm{m}^{3}$ et $7,9 \mathrm{~kg} / \mathrm{m}^{3}$ avaient été obtenues par une station expérimentale située au sein du périmètre et utilisant les mêmes variétés et les mêmes techniques que les agriculteurs.

Dans ces conditions, il peut paraître paradoxal que les performances des périmètres irriguées du Tiers Monde ne soient pas deux à trois fois supérieures et ne se rapprochent pas davantage de celles des pays industrialisés. Cela tient à des causes complexes et éminemment variables d'un pays à l'autre, ou plutôt d'un ensemble régional de pays à l'autre. En conséquence, les solutions doivent être spécifiques et ne peuvent être directement transposées d'un continent à l'autre ; en témoignent, par exemple, les échecs ou les demi-succès de certaines greffes de coopératives ou associations d'irrigants à la mode européenne ou étatsunienne sur des structures paysannes traditionnelles d'Afrique et d'Amérique latine.

Il faut donc adapter les recherches et expérimentations menées en matière de management de l'irrigation à chaque contexte et celles conduites dans les pays industrialisés ne peuvent être que très partiellement utiles aux pays en développement. En sus de conditions agro-climatiques souvent dissemblables, les facteurs handicapant le plus des transferts directs découlent de différences dans les niveaux d'éducation, dans les cultures et dans les structures de coûts.

En dépit de cela, bien peu de pays en développement consacrent des moyens significatifs à l'amélioration du management de leurs périmètres irrigués, surtout en comparaison avec ceux qu'ils continuent à affecter à des travaux de construction ou de réhabilitation. Trop souvent, l'inflation des mètres cubes d'eau stockée, de terre remuée, de volumes transités, etc..., est encore présentée comme un indicateur de développement, indépendamment de l'accroissement de production en résultant. Cette tendance est renforcée par une perception fréquente de la construction d'ouvrages comme une activité noble et rémunératrice contrairement à l'entretien et à la gestion de l'existant. Les sommes à emprunter et le décalage constant entre les bénéfices escomptés et constatés ont rarement été un frein à cette boulimie d'investissement du fait de l'intérêt de la plupart des parties concernées à faire grossir une note dont le remboursement reste presque toujours l'affaire de l'Etat, concept assez flou et souvent lointain. A contrario, les bénéficiaires potentiels d'une gestion plus 
fine de l'eau, que ce soit à l'amont de la parcelle ou dans l'exploitation, répugnent à en payer le prix car celui-ci leur serait directement et immédiatement imputé alors que les gains pouvant en être espérés sont forcément plus lointains et apparaissent souvent comme hypothétiques.

Pourtant, des exemples montrent que des efforts en faveur d'un meilleur management de l'agriculture irriguée peuvent être aussi payants que ceux de la recherche agronomique à l'origine de la "révolution verte" en Asie. Ainsi, les pays d'Europe méditerranéenne doivent une part importante du fulgurant essor de leur agriculture dans les années $60-70$ à un développement volontariste d'une gestion fine des intrants agricoles. Ce constat ne concerne bien évidemment pas que l'eau d'irrigation mais il vaut aussi pour elle.

\section{Mission et modes de travail de I'IIMI}

Les analyses précédentes ont conduit, en 1984, à la création de l'IIMI (Institut international de management de l'irrigation) pour "favoriser le développement, la diffusion et l'adoption d'améliorations durables des performances de l'agriculture irriguée dans les pays en développement ». [II s'agit là de la formulation actuelle de la mission de l'IIMI et non de celle d'origine mais l'esprit en est le même.]

L'IIMI a été créé par un groupe de donateurs comprenant des bailleurs de fonds multilatéraux (Banque mondiale, PNUD, etc...), des gouvernements de pays industrialisés (Australie, Etats-Unis, France, Japon, Pays-Bas et Royaume-Uni) et des fondations privées, la principale étant la Fondation Ford. Bien que le G.C.R.A.I. (Groupe consultatif pour la recherche agronomique internationale) eusse été à l'origine du nouvel institut, il n'a accepté la candidature de l'IIMI qu'en 1991, c'est-à-dire après avoir constaté que ses actions répondaient bien à sa mission et étaient complémentaires de celles des autres membres du groupe.

En fait, l'IIMI a toujours fondé ses actions sur la recherche, la formation et la diffusion de connaissance, comme le préconise le G.C.R.A.I. Cependant cette convergence de méthode ne doit pas masquer que l'objet même de la mission de l'IIMI lui impose de conduire ses activités de manière différente des autres centres du groupe.

En effet, les chercheurs de l'IIMI ne peuvent s'isoler dans des laboratoires ou sur des parcelles expérimentales pour mettre au point les méthodes de management les plus appropriées à un contexte donné car on ne sait pas encore modéliser les réactions humaines au changement, éléments clé pour une amélioration pérenne des performances des périmètres irrigués. De plus, pour permettre à des pays en développement d'entrer durablement dans une dynamique de progrès, il importe moins d'améliorer les performances de leurs périmètres irrigués de l'extérieur que d'induire une capacité locale à adapter celles-ci aux évolutions de leurs contextes physiques, agronomiques, économiques et humains. Ce double constat a conduit l'IIMI à mener toutes ses activités de terrain en liaison étroite avec des agriculteurs, des gestionnaires, des décideurs et des chercheurs des pays concernés. Chaque fois que leurs niveaux de compétence le permettent, l'IIMI laisse d'ailleurs la plus grande part de ses recherches à ses partenaires locaux, se contentant (i) de fournir une méthodologie permettant une valorisation mondiale des résultats, (ii) d'apporter un éclairage international aux investigations menées et (iii) de les aider à surmonter des difficultés inattendues. Ce mode d'action induit un renforcement des compétences des gestionnaires de périmètres concernés et des centres de recherches nationaux et, à terme, cela devrait conduire l'IIMI à ne plus avoir besoin d'intervenir dans les pays ayant le plus bénéficié de ses appuis.

L'IIMI a donc implanté plusieurs antennes en Asie et en Afrique de façon à pouvoir conduire des activités de terrain dans différents contextes en étroite collaboration avec des partenaires nationaux. Ces activités visent à la fois à apporter des solutions à des problèmes locaux et à générer des connaissances de base destinées à alimenter des réflexions plus globales menées par la direction de la Recherche. En conséquence, la moitié de la trentaine de cadres internationaux de l'institut est basée hors du Sri Lanka où se trouvent sa direction générale et son antenne conduisant des recherches dans ce pays et en Inde. Ces cadres "extérieurs" sont installés aux Philippines, au Bangladesh, au Pakistan, au Soudan, au Maroc, au Burkina, au Niger et au Nigeria. Pour compléter cet ensemble, l'institut envisage de créer une antenne en Egypte et prépare un programme pour l'Amérique latine.

Les problèmes liés au management de l'irrigation étant très divers, les moyens limités de l'IIMI ne lui permettent pas de les aborder tous simultanément. Aussi, l'institut a été amené à déterminer des priorités qui s'articulent actuellement autour de cinq programmes * recoupés par quatre thèmes horizontaux **.

Naturellement, les programmes de l'IIMI sont adaptés aux particularismes de chaque région. Ainsi, l'IIMI conseille les pays du sud-est asiatique, menacés par une surproduction de riz, sur les moyens de diversifier leurs cultures. Au Pakistan, pays où la distribution de l'eau est particulièrement rigide et inéquitable, l'institut aide des gestionnaires de périmètres à mieux satisfaire les besoins des agriculteurs, en particulier par l'adoption d'une distribution à la demande. Au Maroc, oủ les faibles efficiences à la parcelle sont la principale cause des rendements modestes des productions irriguées, l'IIMI se concentre principalement sur les conseils aux agriculteurs. En Afrique de l'Ouest, c'est sur les organisations paysannes que l'attention est focalisée du fait de leur rôle primordial dans le management des périmètres irrigués.

* (1) Evaluation et amélioration des performances de l'agriculture irriguée.

(2) Politiques de management sectoriel de l'agriculture irriguée

(3) Amélioration des organismes publics d'irrigation.

(4) Appui au développement du management local de l'irrigation.

(5) Management durable de la fourniture et de l'évacuation des eaux.

** (i) Préservation de l'environnement.

(ii) Choix et bon usage des techniques.

(iii) Rôle des femmes.

(iv) Amélioration des bases de données sur l'agriculture irri- 


\section{Quelques exemples de résultats obtenus par l'IIMI}

Cette dernière partie présente quelques exemples d'interventions de l'IIMI pour illustrer sa philosophie multiforme d'action et montrer le type d'impact qu'il peut avoir dans ses pays d'interventions.

\subsection{Utilisation d'un modèle mathématique pour la gestion d'un canal}

Dès sa création, l'IIMI a été convaincu que les modèles mathématiques sur micro-ordinateurs pouvaient contribuer puissamment à l'amélioration des performances des canaux de transport ou de distribution. En effet, de tels modèles permettent de simuler en quelques jours des années de fonctionnement d'un réseau de canaux tout en produisant un ensemble de données qu'il serait inimaginable d'espérer collecter dans la réalité. Les avantages sont évidents pour tester de nouvelles procédures de régulation, pour former des gestionnaires de canaux ou pour les aider à prendre les décisions les plus appropriées dans des circonstances exceptionnelles. Cependant le réalisme de la simulation des régimes non permanents et la facilité d'utilisation des modèles dépendent des algorithmes utilisés et de la convivialité des interfaces utilisateur.

Aussi, pour mieux évaluer les bénéfices pouvant être tirés de ces modèles, l'IIMI a décidé en 1987 d'en tester les possibilités sur un canal linéaire de transport et de distribution alimentant une partie du périmètre de Kirindi Oya situé au sud du Sri Lanka. Ce canal, d'une trentaine de kilomètres, est coupé d'un grand nombre de régulateurs rendant son pilotage extrêmement délicat. Le CEMAGREF a adapté la présentation d'un de ces modèles non permanent au canal étudié pour en rendre l'utilisation plus facile par les gestionnaires du périmètre. Ces derniers ont vite apprécié l'outil mis à leur disposition car il leur a permis (i) de mieux comprendre la dynamique hydraulique du canal, (ii) d'évaluer a priori les conséquences de manœuvre de régulateurs, (iii) de mettre au point des procédures plus efficaces pour passer d'un état permanent à un autre et (iv) de déterminer des réactions appropriées en cas d'apports pluviaux. Enfin, le modèle a également permis d'évaluer les capacités de transport de chaque bief et, par là-même, de localiser les interventions prioritaires d'entretien.

Fort de ces résultats encourageants, l'IIMI envisage de tester l'utilisation de modèles plus portables que celui utilisé à Kirindi Oya sur d'autres sites et dans d'autres pays.

\subsection{Appuis aux périmètres irrigués gérés par les agricul- teurs}

Le second exemple a trait aux petits périmètres irrigués gérés par les agriculteurs : les PIGA, FMIS en anglais. Ces PIGA peuvent couvrir jusqu'aux deux tiers des surfaces irriguées de certains pays tels que le Népal ou le Maroc. Pourtant, du fait de leurs tailles, de leurs modes de gestion et de l'extrême variabilité de leurs structures, ces PIGA ont reçu moins d'attention des autorités nationales que les grands périmètres, quand ils n'ont pas été complètement ignorés.

L'IIMI a estimé que quelques programmes classiques de recherche-développement ne permettraient pas d'appréhender et de tirer parti de toute la diversité des PIGA. Aussi, il a monté un réseau, le "FMIS network" en anglais, constituant actuellement un lien entre mille trois cents spécialistes des diverses disciplines répartis dans soixante-quinze pays. Ceux-ci peuvent échanger au travers d'un bulletin périodique d'information, confronter leurs expériences lors d'ateliers et influer sur les activités du réseau en participant aux réunions d'un comité d'orientation.

Naturellement, la mise sur pied de ce réseau n'a pas empêché l'IIMI de conduire des recherches sur des PIGA spécifiques quand il y voyait un intérêt particulier. Ainsi, au Maroc, l'IIMI a encouragé un chercheur du département des Sciences humaines de l'Institut agronomique et vétérinaire Hassan II à analyser les modifications du comportement d'irrigants de la vallée de la Tessaout (piémont nord de l'Atlas) après vingt ans de rattachement de leur PIGA au système des grands périmètres irrigués de la plaine du Haouz. Cela a montré que le bouleversement de structures de gestion traditionnelles avait modifié la vision du management des périmètres chez les agriculteurs et pouvait expliquer un niveau de performances plus faible que celui espéré des améliorations physiques apportées aux réseaux traditionnels : revêtement des canaux principaux, régularisation de la ressource, etc..

Il est indéniable qu'après cinq années d'existence, le réseau PIGA a atteint l'un de ses premiers objectifs qui était d'alerter les bailleurs de fonds et les décideurs sur l'existence et l'intérêt des PIGA. De plus, une meilleure connaissance des conditions de fonctionnement de ces petits périmètres a été des plus utiles pour mieux orienter la rétrocession de tout ou partie de la gestion de plus grands périmètres à des organisations paysannes.

\subsection{Conseils à la mise en auvre d'une politique au Sri Lanka}

L'amélioration du management des périmètres irrigués implique, dans la plupart des pays en développement, une modification des appareils législatif et réglementaire les concernant. Or, là comme ailleurs, il n'est généralement pas facile de trouver la meilleure voie et les exemples ne manquent malheureusement pas d'ensembles incohérents et incomplets de mesures gouvernementales étant allés à l'encontre des objectifs visés.

Il paraît évident que des conseils extérieurs ou la connaissance d'expériences étrangères ne peuvent qu'aider des dirigeants à mieux asseoir leurs politiques. Mais il est particulièrement délicat d'intervenir dans ce qui touche à la sphère politique et toute velléité d'«ingérence étrangère " y est souvent suspectée a priori d'atteinte à la souveraineté nationale. Pourtant, l'IIMI s'est risqué à intervenir sur ce terrain glissant au Sri Lanka, avec l'ambition de renouveler les approches des bailleurs de fond bi- ou multilatéraux. 
Le gouvernement sri lankais avait décidé en 1988 de faire participer davantage les irrigants au management des périmètres dans l'espoir de dynamiser et de diversifier l'agriculture irriguée. Mais les responsables ministériels ne savaient trop comment mettre efficacement en œuvre cette politique et l'IIMI s'est proposé de les aider sans chercher à leur imposer quoi que ce soit. L'intervention de l'institut a duré deux ans, de 1990 à 1992, et s'est terminée par la publication d'une série de recommandations approuvées par l'ensemble des parties concernées : quatre ministères, des gestionnaires de périmètres et des représentants d'agriculteurs. De plus, toutes ces parties se sont dites convaincues de la nécessité de mettre rapidement en place les réformes retenues.

Ce consensus entre des groupes dont les intérêts sont traditionnellement considérés comme opposés est un indéniable succès qui augure favorablement de la mise en place de la politique participative voulue par le gouvernement. Cependant, ce résultat n'a pas été acquis facilement puisqu'il a nécessité deux années de discussions, de réflexions et d'interventions d'experts. La difficulté a été bien sûr d'arriver à des compromis sur lesquels pourrait s'établir un consensus, seul mode de prise de décision retenu, dans les cas d'oppositions initiales vives entre les différentes parties. Dans ce processus, l'IIMI n'a joué qu'un rôle de conseiller, de soutien technique et de secrétariat, facilitant les échanges entre les participants sri lankais.

Par-delà le succès représenté par le consensus obtenu et le jugement très positif porté par les Sri Lankais sur l'intervention de l'IIMI, il est encore difficile de savoir si l'impact final des mesures retenues sur les performances des périmètres justifiera l'argent et le temps dépensés dans leur lent processus d'élaboration. L'interrogation est d'autant plus justifiée que le consensus final n'a pu être atteint qu'au prix de compromis éloignés de propositions considérées comme "optimales " par les experts internationaux consultés ; ces mesures consistent en effet essentiellement en des évolutions progressives à partir du système centralisé actuel et non en des transitions plus franches comme ces derniers l'auraient souhaité. Il s'agit donc de savoir si un cheminement détourné, mais accepté par tous, peut permettre une appropriation meilleure et plus rapide du management de périmètres irrigués par les agriculteurs qu'un ensemble de règles plus rationnelles mais qu'il aurait fallu imposer à certains des acteurs essentiels du processus de changement. La réponse ne pourra être donné que dans quelques années.

\section{Conclusion}

Les résultats qui viennent d'être présentés, comme bien d'autres obtenus au cours de ses huit années d'existence, ont convaincu l'IIMI qu'il était possible d'améliorer de façon durable les performances de l'agriculture irriguée du Tiers Monde, tout en garantissant sa rentabilité, en respectant davantage l'environnement et en accroissant la justice sociale. Il n'est certes pas aisé d'avancer dans cette voie et, pour y parvenir, l'IIMI est persuadé qu'il est indispensable de mieux intégrer les aspects techniques et managériaux de l'irrigation. Enfin, l'IIMI croit qu'une capitalisation et une dissémination mondiales des connaissances et des expériences allant dans ce sens peut aussi contribuer à des améliorations notables des performances des périmètres irrigués.

Il est remarquable de constater que cette analyse, paradoxale il y a une décennie, est partagée maintenant par un nombre croissant de pays et d'organisations multilatérales. Cependant, cette prise de conscience ne s'accompagne pas du redéploiement financier requis par l'ampleur et l'urgence de la tâche pour satisfaire les besoins alimentaires d'une population en progression rapide. Cette inadaptation est double : en volume et en nature. Le montant total des crédits de recherche des pays en développement pour l'irrigation et le drainage était estimé à 80 millions de dollars par an en 1990, soit un dix millième des investissements accumulés dans le seul domaine de l'irrigation (et ce qui concernait directement le management de l'irrigation ne représentait qu'une partie de ces crédits !). D'autre part, la plupart des bailleurs de fonds préfèrent financer des projets individualisés répondant à leurs propres préoccupations plutôt que de contribuer à une approche globale et cohérente de l'amélioration du management de l'irrigation dans le Tiers Monde; ainsi les donateurs de l'IIMI imposent d'affecter les trois quarts de leurs dons à des projets particuliers, ce qui ne permet pas à l'institut de valoriser au mieux les enseignements qu'il tire de ses activités de terrain.

Aussi, le formidable défi démographique des prochaines décennies ne pourra être gagné que par un accroissement notable des ressources allouées à la recherche sur le management de l'irrigation dans les pays en développement et par une plus grande concertation internationale sur l'utilisation de ces ressources. 